\title{
Probing the Mechanism of Exocytosis at the Hair Cell Ribbon Synapse
}

\author{
Andreas Neef, ${ }^{1,2 *}$ Darina Khimich, ${ }^{2 *}$ Primoz Pirih, ${ }^{2,3 *}$ Dietmar Riedel, ${ }^{4}$ Fred Wolf, $, 1,5$ and Tobias Moser ${ }^{1,2}$ \\ ${ }^{1}$ Bernstein Center for Computational Neuroscience, Goettingen University, 37073 Goettingen, Germany, ${ }^{2}$ InnerEarLab, Department of Otolaryngology and \\ Center for Molecular Physiology of the Brain, Goettingen University Medical School, 37075 Goettingen, Germany, ${ }^{3}$ Department of Neurobiophysics, \\ University of Groningen, 9747 AG, Groningen, The Netherlands, ${ }^{4}$ Laboratory of Electron Microscopy, Max-Planck Institute for Biophysical Chemistry, \\ 37077 Goettingen, Germany, and ${ }^{5}$ Research Group Theoretical Neurophysics, Department of Nonlinear Dynamics, Max Planck Institute for Dynamics and \\ Self-Organization, 37073 Goettingen, Germany
}

Hearing relies on faithful synaptic transmission at the ribbon synapse of cochlear inner hair cells (IHCs). Postsynaptic recordings from this synapse in prehearing animals had delivered strong indications for synchronized release of several vesicles. The underlying mechanism, however, remains unclear. Here, we used presynaptic membrane capacitance measurements to test whether IHCs release vesicles in a statistically independent or dependent (coordinated) manner. Exocytic changes of membrane capacitance $\left(\Delta C_{\mathrm{m}}\right)$ were repeatedly stimulated in IHCs of prehearing and hearing mice by short depolarizations to preferentially recruit the readily releasable pool of synaptic vesicles. A compound Poisson model was devised to describe hair cell exocytosis and to test the analysis. From the trial-to-trial fluctuations of the $\Delta C_{\mathrm{m}}$ we were able to estimate the apparent size of the elementary fusion event $\left(C_{\text {app }}\right)$ at the hair cell synapse to be $96-223 \mathrm{aF}$ in immature and 55-149 aF in mature IHCs. We also approximated the single vesicle capacitance in IHCs by measurements of synaptic vesicle diameters in electron micrographs. The results (immature, $48 \mathrm{aF}$; mature, $45 \mathrm{aF}$ ) were lower than the respective $C_{\text {app }}$ estimates. This indicates that coordinated exocytosis of synaptic vesicles occurs at both immature and mature hair cell synapses. Approximately $35 \%$ of the release events in mature IHCs and $\sim 50 \%$ in immature IHCs were predicted to involve coordinated fusion, when assuming a geometric distribution of elementary sizes. In summary, our presynaptic measurements indicate coordinated exocytosis but argue for a lesser degree of coordination than suggested by postsynaptic recordings.

Key words: exocytosis; synapse; hair cell; ribbon; sound encoding; fluctuation; capacitance; compound Poisson process

\section{Introduction}

IHCs of the mammalian cochlea encode sound information at their ribbon synapses with auditory nerve fibers (for review, see Fuchs et al., 2003; Nouvian et al., 2006). High rates of transmitter release and of postsynaptic spiking are observed at these synapses. Even brief stimuli evoke exocytosis of multiple vesicles (Moser and Beutner, 2000; Glowatzki and Fuchs, 2002; Brandt et al.,

Received May 2, 2007; revised Sept. 14, 2007; accepted Sept. 21, 2007.

This work was supported by Bernstein Center for Computational Neuroscience Goettingen Federal Department for Education and Research Grant 01GQ0432/01GQ0430 (T.M., F.W.) and by grants from the Deutsche Forschungsgemeinschaft (Center for Molecular Physiology of the Brain), Human Frontier Science Program Organization (RGY0019/2004), and European Commission (Eurohear) (T.M.) A.N. is a Bernstein Fellow at the Bernstein Center for Computational Neuroscience Goettingen. T.M. and F.W. designed the research and contributed to analysis and modeling. D.K. performed the patch-clamp recordings and contributed to analysis by segment selection. D.R. performed the electron microscopy and measured vesicles diameters. P.P. initiated and performed analysis and modeling. A.N. completed analysis methodology, performed analysis and modeling, and tested it extensively on surrogate data. T.M., A.N., and F.W. wrote this manuscript. We thank E. Neher, M. Holt, and S. Young for comments on this manuscript and M. Köppler for excellent technical assistance.

*A.N., D.K., and P.P. contributed equally to this work.

Correspondence should be addressed to either of the following: Tobias Moser, InnerEarLab, Department of Otolaryngology, University of Goettingen, Center for Molecular Physiology of the Brain, Bernstein Center for Computational Neuroscience, 37099 Goettingen, Germany, E-mail: tmoser@gwdg.de; or Fred Wolf, Research Group Theoretical Neurophysics, Department of Nonlinear Dynamics, Max Planck Institute for Dynamics and SelfOrganization, Bunsen-Strasse 10, 37073 Goettingen, Germany, E-mail: fred-wl@nld.ds.mpg.de.

DOI:10.1523/JNEUROSCI.1996-07.2007

Copyright $\odot 2007$ Society for Neuroscience $\quad$ 0270-6474/07/2712933-12\$15.00/0
2005; Griesinger et al., 2005; Khimich et al., 2005). The occurrence of kinetically uniform but variably sized postsynaptic currents indicated that several vesicles can be released with high synchrony at hair cell synapses (Glowatzki and Fuchs, 2002; Keen and Hudspeth, 2006). However, the underlying mechanism is still unclear. Homotypic fusion of ribbon-tethered vesicles to each other, e.g., triggered by a sufficiently strong $\mathrm{Ca}^{2+}$ signal at the active zone, has been suggested as a possible mechanism underlying coordinated multivesicular release at the ribbon synapse (Heidelberger et al., 1994; Glowatzki and Fuchs, 2002; Lenzi et al., 2002; Parsons and Sterling, 2003; Edmonds et al., 2004; Spassova et al., 2004). Multivesicular release also occurs at conventional synapses (Tong and Jahr, 1994; Auger et al., 1998; Llano et al., 2000; Wadiche, 2001; Oertner et al., 2002) and may be coordinated by $\mathrm{Ca}^{2+}$ induced $\mathrm{Ca}^{2+}$ release (CICR) (Llano et al., 2000). CICR has also been indicated to contribute to the synaptic $\mathrm{Ca}^{2+}$ signaling in inner hair cells (Kennedy and Meech, 2002).

Here, we explored the mechanism of exocytosis underlying hair cell transmitter release using membrane capacitance measurements $\left(C_{\mathrm{m}}\right)$ in mouse IHCs before and after the onset of hearing. Although whole-cell $C_{\mathrm{m}}$ measurements have been instrumental for characterizing exocytosis occurring across all synapses of a hair cell (for review, see Nouvian et al., 2006), they cannot directly resolve the fusion of an individual synaptic vesicle 
because of the small vesicle capacitance. To explore the microscopic properties of the release process we took advantage of the quantal nature of transmitter release (Del Castillo and Katz, 1954), which contributes to trial-to-trial fluctuations among exocytic whole-cell capacitance increments during repetitive stimulation. Analysis of such fluctuations can reveal the apparent size of elementary fusion events $\left(C_{\text {app }}\right)$. If vesicles fuse independent of each other this estimate is identical to the average vesicle capacitance, as in the case of chromaffin granules (Moser and Neher, 1997a). However, if release of vesicles was coordinated (statistically dependent) the apparent elementary size would be larger than the capacitance of a single vesicle. We obtain estimates of the mean apparent size of the fusion events in immature and mature IHCs and compare them to estimates of single synaptic vesicle capacitance that we derived independently by electron microscopy of the IHC synapses. Our results indicate that coordinated release occurs at both mature and immature synapses but seems not to involve CICR.

\section{Materials and Methods}

Patch-clamp recordings. Wild-type mice (Naval Medical Research Institute) with postnatal ages of $7-9$ or $14-20 \mathrm{~d}$ were killed by decapitation in accordance with the animal welfare guidelines of the University of Goettingen. The cochlea was dissected in HEPES Hank's solution containing (in mM) $5.36 \mathrm{KCl}, 141.7 \mathrm{NaCl}, 1 \mathrm{MgCl}_{2}, 0.5 \mathrm{MgSO}_{4}, 0.1 \mathrm{CaCl}_{2}, 10 \mathrm{Na}$ HEPES, $10 \mathrm{D}$-glucose, and $3.4 \mathrm{~L}$-glutamine. IHCs from the apical coils of freshly dissected organs of Corti were patch-clamped at their basolateral face at room temperature $\left(20-25^{\circ} \mathrm{C}\right)$ in the perforated-patch configuration as described previously (Brandt et al., 2003). The Pipette solutions contained the following (in $\mathrm{mM}$ ): $I_{1}, 150 \mathrm{~K}$-gluconate, $2 \mathrm{MgCl}_{2}, 10 \mathrm{~K}$ HEPES; $I_{2}: 150$ Cs-gluconate, $2 \mathrm{MgCl}_{2}, 10 \mathrm{Cs}$-HEPES. The extracellular solutions contained (in mM) $105 \mathrm{NaCl}, 35$ tetraethylammonium-Cl, 2.8 $\mathrm{KCl}, 10 \mathrm{CaCl}_{2}$ (or $1.3 \mathrm{CaCl}_{2}$ in a subset of experiments), $1 \mathrm{MgCl}_{2}, 10$ $\mathrm{Na}$-HEPES, and $10 \mathrm{D}$-glucose. In some experiments, $100 \mu \mathrm{M}$ ryanodine was added. All solutions were adjusted to $\mathrm{pH} 7.2$ and had osmolarities between 300 and $320 \mathrm{mmol} / \mathrm{L}$. Shortly before experiments $250 \mu \mathrm{g} / \mathrm{ml}$ Amphotericin B were mixed into the intracellular solution by sonication. The chemicals were obtained from Sigma (St. Louis, MO) except for Amphotericin B (Calbiochem, La Jolla, CA). Cs-gluconate salt was made from $\mathrm{CsOH}$ and $\mathrm{D}$-gluconic acid solutions.

Data acquisition and analysis. EPC-9 amplifiers (HEKA Electronics, Lambrecht, Germany) controlled by "Pulse" software (HEKA Electronics) were used for measurements and data acquisition. Currents were sampled at $20 \mathrm{kHz}$ and low-pass filtered at $2 \mathrm{kHz}$. We measured $C_{\mathrm{m}}$ using the Lindau-Neher technique (Lindau and Neher, 1988), implemented in the software-lockin module of "Pulse". A $1 \mathrm{kHz}, 70 \mathrm{mV}$ peak-to-peak sinusoid was applied about a direct current (DC) holding potential of $-84 \mathrm{mV}$. All voltages were corrected for liquid junction potential $(-14$ $\mathrm{mV}$ ). For the impedance analysis the reversal potential of the DC current was set to the reversal potential of the slow tail currents after the depolarizations as described previously (Moser and Beutner, 2000). IHCs were stimulated by 10 or $20 \mathrm{~ms}$ step depolarizations to the cell's peak $\mathrm{Ca}^{2+}$ current potential (mostly between -10 and $5 \mathrm{mV}$ ) and by dummy stimuli ( $-84 \mathrm{mV}$ for the same duration). Each $C_{\mathrm{m}}$ sweep was $12 \mathrm{~s}$ long and contained one depolarization and two dummy stimuli (see Fig. 3). For the analysis, we used the first dummy stimulus for all but four sweeps. We used interstimulus intervals from 12 to $32 \mathrm{~s}$ in different cells, which is longer than the $90 \%$ refilling time of the readily releasable pool (RRP) $(<10 \mathrm{~s})$ (Moser and Beutner, 2000).

Electron microscopy. Mouse organs of Corti were fixed immediately after dissection with 2.5\% glutaraldehyde in HEPES-Hanks solution (see above) for $30 \mathrm{~min}$ at room temperature. Thereafter, the samples were fixed overnight at $4^{\circ} \mathrm{C}$ in $2 \%$ glutaraldehyde in $0.1 \mathrm{M}$ cacodylate buffer at $\mathrm{pH}$ 7.4. After an additional fixation in $0.1 \% \mathrm{OsO}_{4}$, the samples were stained with $1 \%$ uranyl acetate, dehydrated in a series of EtOH solutions, and finally soaked in propylene oxide. They were then embedded in Agar 100 (purchased from Science Services, Munich, Germany). Thin sections
$(80 \mathrm{~nm})$ were counterstained with lead citrate and examined using a Philips CM 120 BioTwin transmission electron microscope (Philips, Eindhoven, Netherlands). Pictures were taken with a TemCam F224A camera (TVIPS, Gauting, Germany) at 27,400-fold magnification. The microscope was calibrated using a catalase crystal calibration specimen. Because the ribbon associated membrane profiles were slightly elliptical, the longest and shortest diameters (outer rim of outer leaflets) of each profile were measured with Digital Micrograph 3.4 software (Gatan, Munich, Germany) and averaged to calculate the mean vesicle diameter. To avoid underestimation of the vesicles diameter by capping (imaging of small portions of a sectioned vesicle), only profiles with clearly delineated membranes were measured, maximizing the chances of reporting the largest diameter.

Data analysis and modeling. Data analysis and modeling were performed in Matlab (Mathworks, Natick, MA) and Igor Pro (Wavemetrics, Eugene, OR) software. Means are presented with their SE.

Modeling hair cell release as a compound Poisson process. We modeled evoked $\Delta C_{\mathrm{m}}$ values with a compound Poisson process. In such a process, the number $K$ of statistically independent events during an interval $T$ follows a Poisson distribution with a rate $\lambda$, whereas the sizes $r_{i}$ of those events follow a known distribution $P(R)$. The sum $S$ of the $k$ events $r_{i}$ that occurred during $T$ is given as follows:

$$
s=\sum_{i=1}^{k} r_{i}
$$

It is said to follow a compound Poisson distribution $(\mathrm{CP})$ and has the expectation value

$$
E(S)=\lambda \mathrm{T} \times \mathrm{E}(R)
$$

and the variance

$$
\operatorname{var}(S)=\lambda \mathrm{T} \times \mathrm{E}(R)^{2}+\lambda T \times \operatorname{var}(R) .
$$

A derivation for the more general case of two combined distributions is given in the Appendix. In our experiments, $k$ individual fusion events occur during the stimulus duration $T$. However, we can only observe the realizations $s_{i}$ as evoked capacitance changes $\Delta C_{\mathrm{m}}$. From the analysis, we obtain an estimate for $\operatorname{var}(S) / E(S)$ that we call apparent elementary size $\left(C_{\text {app }}\right)$ :

$$
C_{a p p}=\mathrm{E}(R) \times\left(1+C V^{2}\right) .
$$

It depends on the average size of a release event $E(R)$ and the coefficient of variation $(\mathrm{CV})$ of the size distribution $P(R)$.

Let us consider the special case where the elementary release events are discrete multiples of a minimal quantum, the synaptic vesicle (SV) capacitance $\left(C_{\mathrm{sv}}\right)$, and those multiples follow a geometric distribution, $P(R=r)=\mu^{-1}\left(1-\mu^{-1}\right)^{\mathrm{r}-1}$, with the mean $\mu$ and the variance $\sigma^{2}=$ $\mu\left(1-\mu^{-1}\right)$. Then, Equation 4 simplifies to the following:

$$
C_{a p p}=C_{s v} \times[2 \mu-1] .
$$

The interpretation of the measurements is guided by this relation. However the general form given in the appendix (Equation A11) can be used to derive similar equations for other special compound processes.

Data analysis. The analysis from raw $C_{\mathrm{m}}$ data to apparent capacitance of the elementary release event involved several steps, which are detailed in the following sections. The fidelity and reliability of the entire analysis was studied by analyzing simulated realistic $C_{\mathrm{m}}$ traces for which the "true" size distribution of the elementary release events is known.

Data import and segment selection. Capacitance traces were imported from Pulse software and processed in an interactive custom analysis software programmed in Matlab. During this step, the amount of data were decimated by averaging each two adjacent points. To avoid the contribution of glitches as well as excess low or high frequency noise in the $C_{\mathrm{m}}$ segments, we selected the longest possible, reliable, and low noise stretch of each $C_{\mathrm{m}}$ segment. To exclude bias in this selection, segments were presented in random order and always oriented "away" from the stimulus (depolarization or dummy stimulus) (i.e., segments recorded 
before a stimulus were presented time-reversed). Therefore, the investigator had no information about the nature of the stimulus (depolarization or dummy stimulus) and the position of the segment (preceding or following the stimulus).

Capacitance step estimation. The selected regions before and after each stimulus (depolarization or dummy stimulus) were joined and fit with the sum of a linear function (overall trend) and a Heaviside-function (step). The use of more versatile fit functions with either an additional poststimulus trend (linear or single exponential) did not increase the quality of the fit. A triplet of $\Delta C_{\mathrm{m}}$ values was obtained from each sweep: one evoked (depolarization) and two spontaneous (dummy stimuli). In all but four sweeps, the first of the spontaneous $\Delta C_{\mathrm{m}}$ values was used for analysis. In those four instances, the second value replaced the first because this was detected to be an outlier (see below, Calculation of confidence intervals).

Nonstationary fluctuation analysis. Because of rundown of the exocytic responses, we used nonstationary noise analysis. This involved comparison of $\Delta C_{\mathrm{m}}$ values within short time windows (ensemble of five sweeps), for which we assumed the response to be stationary. Five series of these nonoverlapping windows were used. Furthermore, for correct variance estimation, we removed the trend resulting from the rundown (Moser and Neher, 1997a). The trend was described by a Double-exponential fit to the $C_{\mathrm{m}}$ values or by forward-reverse low-pass filtering of the $C_{\mathrm{m}}$ values (filtfilt function in Matlab). The used finite-impulse-response filter kernel had a cutoff frequency of 0.1 sample $^{-1}$ and an order of 10 (i.e., the 10 last data points influence the filtering). The estimate for the variance/ mean ratio $\left(C_{\text {app }}\right)$ has been obtained by a line fit to the variances and means resulting from the noise analysis (Fig. 5). The inclusion of the data from dummy stimulations ascertained that the considerable spontaneous $\Delta C_{\mathrm{m}}$ fluctuations did not affect our estimates. When we tested the influence of the low-pass cutoff frequency on $C_{\text {app }}$, it turned out that for sufficiently low values (below 0.2 sample $^{-1}$ ) the resulting $C_{\text {app }}$ did only weakly depend on the cutoff frequency showing that the separation between the slower trend and the fast intertrial fluctuations was possible.

Calculation of confidence intervals. Nonparametric estimates of the $95 \%$ confidence interval (CI) for $C_{\text {app }}$ were obtained by bootstrap. We used this method because the variability of the mean declined with the mean and therefore the dataset is heteroscedastic. In a bootstrap statistics the available variance/mean data are treated like an underlying population. A random sample is drawn from them (with replacement) and the statistic of interest (i.e., the slope $C_{\text {app }}$ is calculated). Over many (in our analysis over 500) repetitions of random resampling a bootstrap distribution of $C_{\text {app }}$ values develops. The 2.5 and $97.5 \%$ percentiles of this distribution are the limits of the estimated CI. The same approach was used to combine the $C_{\text {app }}$ values of the individual cells within each group. Here, bootstrapping was used to estimate the $\mathrm{CI}$ of the grand average for each group.

Less than $2 \%$ of the $\Delta C_{\mathrm{m}}$ value pairs (evoked and corresponding spontaneous) were excluded as outliers. They were detected fully automated with jackknife. Depending on the applied trend correction (low-pass filtering or double exponential fit) 47 or 65 of $4058 \Delta C_{\mathrm{m}}$ pairs (spontaneous and evoked $\Delta C_{\mathrm{m}}$ ) were detected as outliers. The method is based on a jackknife-derived statistical analysis of the bootstrap CI where any one $\Delta C_{\mathrm{m}}$ value pair was removed from the dataset. If removal of a given $\Delta C_{\mathrm{m}}$ value pair reduced the bootstrap CI by $20 \%$, it was rejected from the analysis. The exclusion process was iterated until the $80 \%$ criterion did not detect any more outlier.

Extreme values of spontaneous $\Delta C_{\mathrm{m}}$ in some cases did not affect the CI of the estimated $C_{\text {app }}$ but its absolute value. Those outliers were detected by their influence on the average variance of spontaneous $\Delta C_{\mathrm{m}}$, which, therefore, was used as reference. If exclusion of a data pair shifted the variance by more than five SDs from the reference, the respective spontaneous $\Delta C_{\mathrm{m}}$ was considered an outlier and removed together with its corresponding evoked $\Delta C_{\mathrm{m}}$. In four of these cases it was possible to replace the outlying value by the $\Delta C_{\mathrm{m}}$ of the second dummy stimulus.

Simulating realistic $\mathrm{C}_{m}$ traces. We thoroughly tested the analysis steps using simulated $C_{\mathrm{m}}$ traces. They consisted of a step function (describing the exocytic $C_{\mathrm{m}}$ increment; step sizes follow the compound Poisson release model with underlying geometric distribution as described above) superimposed with Gaussian noise $G_{\mathrm{n}}$ (to resemble the dominating Johnston noise of the recording configuration) and a slowly varying drift term $D$ [Ohrnstein-Uhlenbeck process (oup)], mimicking the slow $C_{\mathrm{m}}$ drifts observed in the experiments. The drift term was generated as a time series with an interval $\Delta t$ of $2 \mathrm{~ms}$ :

$$
D(t+\Delta t)=D(t) \cdot e^{\frac{t}{\tau}}+G_{\text {oup }}\left(\mu=0, \sigma_{\text {oup }}^{2}\right) .
$$

To match the power spectra of the simulated and real $C_{\mathrm{m}}$ traces, the Gaussian noise $G_{\text {oup }}$, driving the Ohrnstein-Uhlenbeck process, had to have an $\mathrm{SD} \sigma_{\text {oup }}$ between 0.13 and $1.3 \mathrm{fF}$ and a $\tau$ of $250 \mathrm{~ms}$, whereas the additional Gaussian $G_{\mathrm{n}}$ noise that was added to the series $D(t)$ had SDs $\sigma_{n}$ from 3 to $6 \mathrm{fF}$ :

$$
\text { Noise }(t)=D(t)+G\left(\mu=0, \sigma_{n}^{2}\right)
$$

To simulate a dummy stimulus trace, $25 \mathrm{~ms}$ were cut out of a noise trace; no step was added. To yield realistic $\Delta C_{\mathrm{m}}$ values for a whole experiment $\lambda$ decayed exponentially with the sweep number. For instance, we used $\mu=2, C_{\mathrm{ves}}=50 \mathrm{aF}$ with $\lambda$ starting at an initial value of $100-300$ decaying to final values between 30 and 150 with time constants of 5-60 sample periods. The length of the simulated experiments was varied from 50 to 300 sweeps.

Simulated data were used for two purposes. First, the whole analysis from step estimation to bootstrap was tested. It turned out that the model parameter $\mu$ was recovered by the analysis very well with a small bias toward smaller values. Second, the simulated data were used to assess the influence of weighting when combining the results of multiple experiments.

\section{Results}

\section{RRP exocytosis in response to stimulation: model and experiment}

Postsynaptic recordings have indicated highly synchronized release of multiple synaptic vesicles from hair cells (Glowatzki and Fuchs, 2002; Keen and Hudspeth, 2006), suggesting statistical interdependence of vesicle exocytosis. To approximate the statistics of these EPSCs and to aid our analysis, we developed a compound Poisson model of vesicle release. It describes the occurrence of independent release events with a rate $\lambda$ whereas the sizes of the release events themselves can follow an arbitrary distribution (see Materials and Methods) (for a general treatment, see the Appendix). In this way, the model can accommodate various degrees of statistical interdependence of synaptic vesicle exocytosis. For instance, if the size distribution of the elementary events had a mean of one $(\mu=1)$ with zero variance, it would represent a Poisson driven release of single vesicles (no coordination) (Fig. $1 A$, left).

Postsynaptic recordings of EPSC at IHC synapses, indeed, observed an exponential interevent interval distribution characteristic of a Poisson process, whereas the EPSC distribution was reasonably well approximated with geometric distributions. As an example we reproduced one of the EPSC distributions from Glowatzki and Fuchs (2002) in Figure $1 B$, which was fitted well with a $\mu$ of four suggesting that at this synapse on average four synaptic vesicle equivalents were released within a single exocytic event. This agrees well with the average values of mean EPSC amplitude (148 pA) and putative miniature EPSC amplitude (30-36 pA) reported by Glowatzki and Fuchs (2002). These two experimental findings suggest that a Poisson process that drives of elementary release events with geometrically distributed sizes might adequately describe exocytosis at IHC synapses. The underlying size distribution of elementary release events can be characterized using estimates of ensemble variance and mean obtained from repetitively evoked exocytic responses (Fig. 1C).

Here we monitored IHC exocytosis as increment of mem- 
brane capacitance $\left(\Delta C_{\mathrm{m}}\right)$ by repetitive stimulation obtained many trials (see below). Under these conditions only a fraction of the trial to trial changes in the exocytic response is caused by the quantal nature of the release process. It is occluded by a much larger measurement noise and a long term trend (rundown, which likely reflects a decrease in release rate $\lambda$ ). To estimate the variability that correlates with the rate of release, we characterized the measuring noise during dummy stimuli (during which the release rate $\lambda$ is zero) and corrected for the trend. We can then calculate the apparent capacitance of an elementary fusion event $\left(C_{\text {app }}\right)$ from $\Delta C_{\mathrm{m}}$ ensemble estimates of variance and mean by linear regression (Materials and Methods) (Fig. $5 B$ ). Once $C_{\text {app }}$ and the "true" capacitance of a single synaptic vesicle $\left(C_{\mathrm{sv}}\right)$ in hair cells are known, we can evaluate the statistical interdependence of synaptic vesicle fusion. If $C_{\text {app }}$ exceeds $C_{\mathrm{sv}}$ this indicates that vesicles do not fuse in a statistically independent manner.

\section{Approximating the single synaptic vesicle capacitance from electron microscopy}

To obtain an estimate of the $C_{\mathrm{sv}}$ for comparison with the result of the noise analysis $\mathrm{C}_{\text {app }}$, we used transmission electron microscopy. We measured synaptic vesicle diameters in $80 \mathrm{~nm}$ sections of mouse organs of Corti at postnatal day 8 (before the onset of hearing, four cochleae of two mice) and at postnatal days 14-15 (after the onset of hearing, nine cochleae of five mice). Apical cochlear coils were rapidly explanted as for electrophysiology and immediately transferred into fixative without any intentional stimulation of the IHCs (low $\mathrm{Ca}^{2+}$ solution) (see Materials and Methods). Figure 2 shows representative electron micrographs of active zones of immature $(A)$ and mature $(B)$ IHCs.

Most membrane profiles tethered by the synaptic ribbon (ribbon-associated) took a near spherical shape (slightly elliptical) and homogeneous small size as expected for synaptic vesicles (for a recent quantitative description of CNS synaptic vesicles see Takamori et al., 2006). We did not systematically observe larger sized ribbon-associated membrane profiles in our material. In addition to nonribbon-associated synaptic vesicles, we observed clathrin-coated endocytic vesicles as well as larger, variably shaped uncoated membrane profiles in the cytosol surrounding the ribbon in both immature and mature IHCs.

We estimated the outer diameter of ribbon-associated membrane profiles (e.g., see inset in Fig. 2 A, Materials and Methods section for details) from which we subtracted the mean membrane thickness $(6.2 \pm 0.8 \mathrm{~nm} ; n=56 \mathrm{SV})$ to yield the midmembrane diameter relevant for the calculation of the membrane capacitance added after vesicle fusion $\left(\mathrm{C}_{\mathrm{sv}}\right)$. The resulting diameter distributions for ribbon-associated membrane profiles of immature and mature IHCs were nearly Gaussian (Fig. 2C) with means
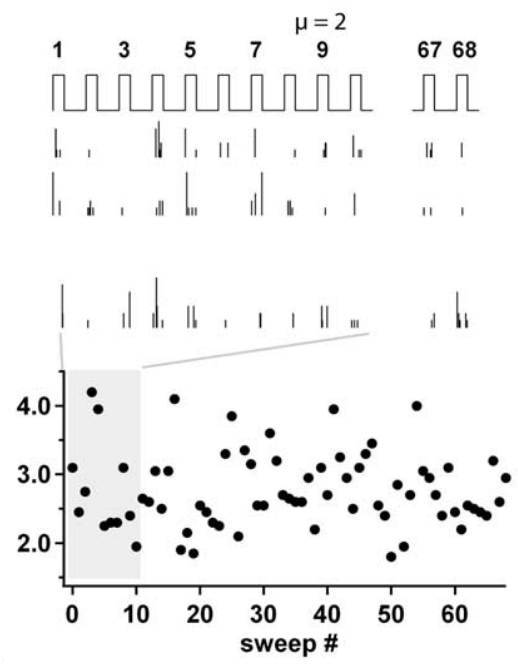

C

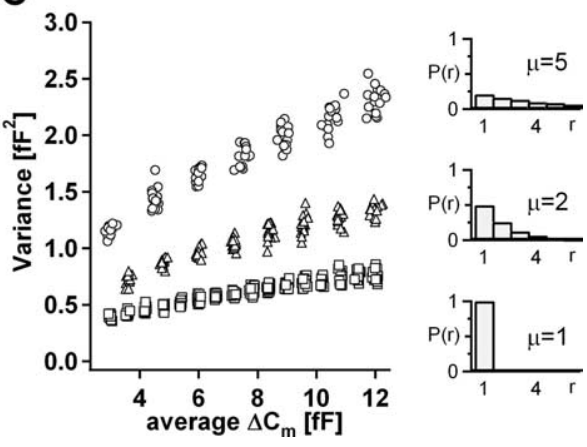

Figure 1. Interpreting hair cell exocytosis based on a Compound Poisson model of release. $A$, Realizations of this model for 12 individual synapses (top trace, stimulus timing). For simplicity, the release probability was scaled to match the average cumulative (

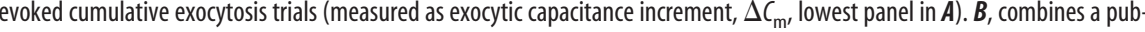
scatterplot produced by realizations of the compound Poisson models with different values for $\mu: 1,2$ (as in $\boldsymbol{A}$ ) and 5 at different release rates $\lambda$ and their corresponding geometric distributions (histograms).

of $38.4 \pm 0.5 \mathrm{~nm}(\mathrm{CV}, 17 \% ; n=602)$ and $36.9 \pm 0.6 \mathrm{~nm}(\mathrm{CV}$, $20 \% ; n=574)$. For comparison, we included the result of an identical analysis of CNS synaptic vesicles expected to show no shrinkage because they were high pressure-quick frozen and imaged on a cryo-electron microscope (Fig. 2C, dashed line) (Takamori et al., 2006). This distribution was not significantly different from the one of ribbon-associated membrane profiles in chemically fixed mature IHCs $(p=0.84)$. It neither differed from the distribution of CNS synaptic vesicles obtained from brain slices of mouse hippocampus or cultured hippocampal cells obtained after chemical fixation and embedding in Epon (data not shown). The vesicle diameter distributions were transformed into capacitance distributions (Fig. 2D) assuming a spherical shape and a specific membrane capacitance of $10 \mathrm{fF} \mu \mathrm{m}^{-2}$ (Breckenridge and Almers, 1987; Gentet et al., 2000). The average $C_{\mathrm{sv}}$ amounted to $47.7 \pm 0.7 \mathrm{aF}(\mathrm{CV}, 0.34)$ for immature IHCs and $44.5 \pm 0.8 \mathrm{aF}$ $(\mathrm{CV}, 0.43)$ for mature IHCs.

Estimation of $C_{\text {app }}$ from analysis of trial-to-trial fluctuations Whole-cell $C_{\mathrm{m}}$ measurements of the mean capacitance added to the IHC plasma membrane by an individual release event present a substantial challenge. The capacitance of mouse IHCs $(\sim 7.5 \mathrm{pF}$ 

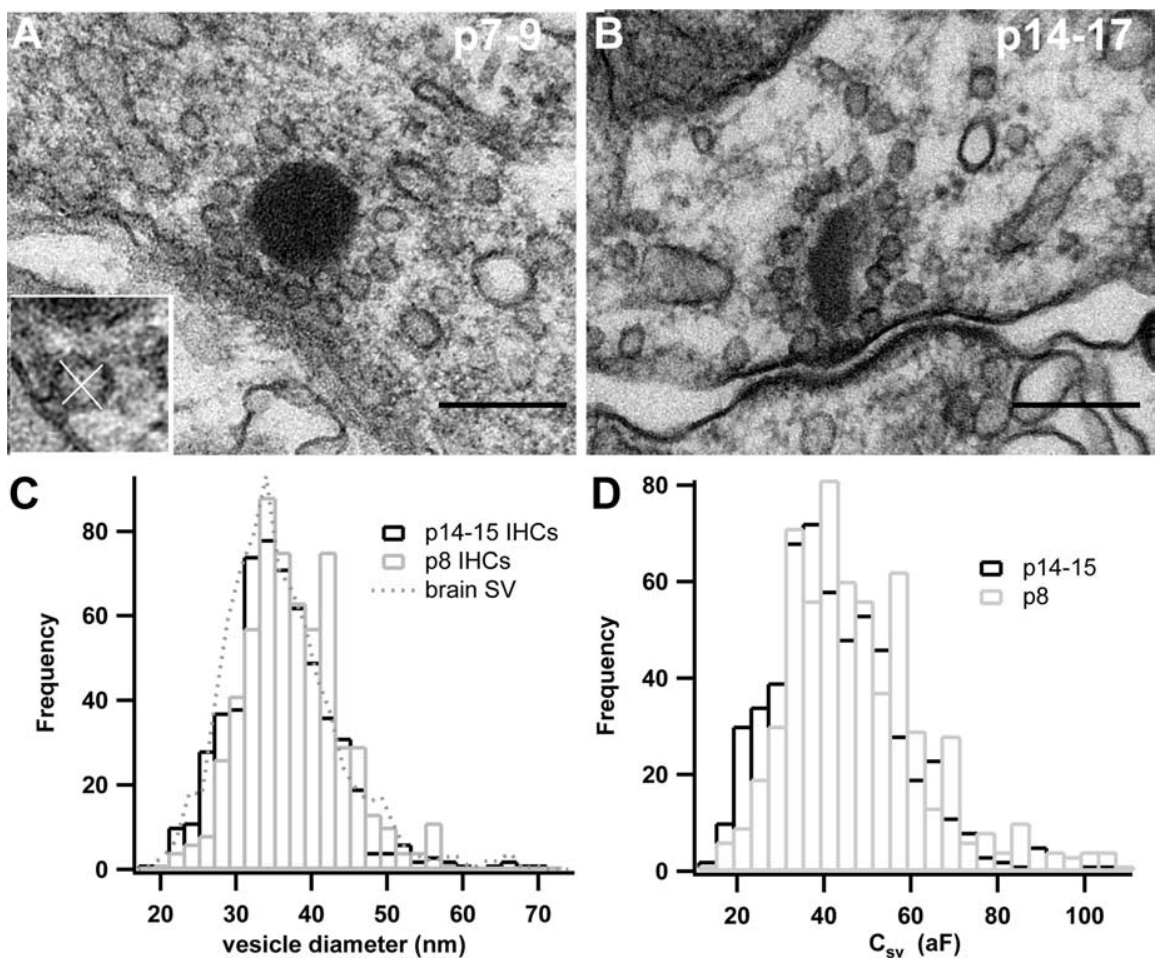

Figure 2. Approximation of the mean single synaptic vesicle capacitance, $C_{s v} \cdot \boldsymbol{A}, \boldsymbol{B}$, Representative electron micrographs of immature and mature ribbon-type active zones (scale bar, $200 \mathrm{~nm}$ ). Whereas the immature synapse features round (and often multiple) dense bodies, a single, more ovoid ribbon is typical for the mature IHC synapse. Inset, The orthogonal gray lines illustrate the measurement of the vesicle diameter. $\boldsymbol{C}$, The distribution of the estimated midmembrane diameters for immature (gray) and mature (black) IHCs after chemical fixation and for cryo-EM-imaged synaptic vesicles purified from the rat brain (dashed line) (data from Takamori et al., 2006). We presently do not have an explanation for the high frequency of diameters in the range of $42-45$ $\mathrm{nm}$ found in the immature IHCs. D, represents the distributions of our $C_{\mathrm{sv}}$ estimates obtained from transformation of the diameter distributions $\left(\pi \mathrm{d}^{2} \times 10 \mathrm{fF} \mu \mathrm{m}^{-2}\right)$.

Table 1. Passive electrical properties

\begin{tabular}{lllll}
\hline & $n_{\mathrm{IHCs}}$ & $C_{\mathrm{m}}(\mathrm{pF})$ & $R_{\mathrm{s}}(\mathrm{M} \Omega)$ & $I_{\text {rest }}(\mathrm{pA})$ \\
\hline Immature IHCs & $n=10$ & $7.3 \pm 0.2$ & $16.0 \pm 1.0$ & $-17 \pm 4.1$ \\
Mature IHCS & $n=14$ & $7.9 \pm 0.3$ & $14.7 \pm 1.1$ & $-6.9 \pm 2.4$
\end{tabular}

The table presents grand averages ( \pm SEM) of the mean $I_{\text {rest }}$ (at the holding potential of $-84 \mathrm{mV}$ ), $C_{\mathrm{m}}$ and $R_{\mathrm{s}}$ of a sample of immature and mature IHCs recorded with pipette solution $I_{1} \cdot I_{\text {rest }} C_{\mathrm{m}}$ and $R_{\mathrm{s}}$ were tracked during the recording as the average values obtained for each sweep.

in the present study) exceeds $C_{\mathrm{sv}}$ by more than five orders of magnitude. In addition to this huge baseline capacitance, other biological and technical noise sources such as thermal noise of the access resistance to the cell have to be taken into consideration. As this approach pushes the envelope of whole-cell $C_{\mathrm{m}}$ measurements, we optimized the recordings and controlled the precision and reliability of the fluctuation analysis by accompanying modeling.

Low noise-recordings of whole-cell $C_{\mathrm{m}}$ and $\Delta C_{\mathrm{m}}$ estimation To minimize capacitance noise we used large $(70 \mathrm{mV}$ peak-topeak) sinusoidal voltage commands. We did not find evidence for $\mathrm{Ca}^{2+}$ channel activation or inactivation by the large sinusoidal voltage command (data not shown). The perforated-patch configuration permitted long-lasting recordings of up to 480 exocytic responses with stable and large-conductance access to the cell (for passive electrical properties of the cells, see Table 1). In addition, we recorded long $C_{\mathrm{m}}$ stretches before and after the depolarizing stimulus (up to $3 \mathrm{~s}$ of $C_{\mathrm{m}}$ before and after the stimulus were used in the analysis) assuming that exocytosis predominantly occurs synchronized with the stimulus and that endocytosis is comparatively slow (Moser and Beutner, 2000; Beutner et al., 2001). Figure $3 A$ displays a typical $C_{\mathrm{m}}$ measurement recorded from a mature IHC along with the corresponding membrane and series conductance traces $\left(G_{\mathrm{m}}\right.$ and $\left.G_{\mathrm{s}}\right)$. The pulse protocol indicates the $20 \mathrm{~ms}$ depolarizing stimulus and the two dummy stimuli that allowed us to assess the stimulus-independent $C_{\mathrm{m}}$ variance resulting from to the cell and the recording system. Thus, each $C_{\mathrm{m}}$ sweep provided a triplet of one evoked and two spontaneous $\Delta C_{\mathrm{m}}$ estimates. Another sample of IHCs was stimulated by a protocol in which the depolarization (at nine seconds) followed two dummy stimuli (separated by three seconds), aiming to gather a still longer baseline before the depolarization for most faithful subtraction of trends. However, both stimulation paradigms resulted in indistinguishable estimates of the apparent elementary size (data not shown) and were therefore pooled.

The $C_{\mathrm{m}}$ traces showed a transient change during the first milliseconds after the depolarization $(\tau \sim 30 \mathrm{~ms})$, probably reflecting a $G_{\mathrm{m}}$ change that contaminated the $C_{\mathrm{m}}$ and $G_{\mathrm{s}}$ estimates (Fig. $3 A, C$ ). It is present also when exocytosis is abolished by blocking $\mathrm{Ca}^{2+}$ influx [Brandt et al. (2003), their Fig. 3B]. It most likely reflects a bleed through of a membrane conductance change and can be reduced by appropriate settings of the "sine $+\mathrm{dc}$ " lock-in estimation (Moser and Beutner, 2000). Therefore, we discarded the first $40 \mathrm{~ms}$ to safely isolate the exocytic $C_{\mathrm{m}}$ increment. Figure $3 B, C$ show average $C_{\mathrm{m}}$ traces of 12 mature $(B)$ and 7 immature $(C)$ IHCs that conformed to the paradigm described in Figure $3 A$. The traces demonstrate stable baselines with a negligible negative $C_{\mathrm{m}}$ trend of $\sim 1 \mathrm{fFs}^{-1}$ that was comparable before and after the depolarization (different by less than a factor of two). Hence, endocytosis did not cause any major reduction of our $\Delta C_{m}$ estimates. We observed a general trend of $C_{\mathrm{m}}$ decline in our perforated-patch recordings from immature and mature IHCs, which is presently not understood.

To further minimize and quantify the error of our final $\Delta C_{\mathrm{m}}$ measurement, we applied a number of procedures. (1) We performed a "blinded" selection for the most reliable $C_{\mathrm{m}}$ segments (see Materials and Methods) to exclude occasional glitches in the $C_{\mathrm{m}}$ traces. (2) Each of the three sets of selected $C_{\mathrm{m}}$ segments was approximated by a nested linear model (model fit in Fig. $3 A$ ) (see Materials and Methods) to yield basal $C_{\mathrm{m}}$ the $\Delta C_{\mathrm{m}}$ step and the overall $C_{\mathrm{m}}$ trend. (3) To test the validity of $\Delta C_{\mathrm{m}}$ estimation, we generated artificial $C_{\mathrm{m}}$ traces using the above described model of exocytosis and simulated temporal fluctuations in $C_{\mathrm{m}}$ by a superposition of Gaussian Noise and an Ohrnstein-Uhlenbeck process to match the noise properties of the data (see Materials and Methods). The lowest panel in Figure $3 A$ illustrates a typical artificial $C_{\mathrm{m}}$ trace and the corresponding model fit used to estimate test and spontaneous $\Delta C_{\mathrm{m}}$. These simulations allowed us to scrutinize analysis and error of the final $C_{\text {app }}$ estimate (see below). 


\section{Rundown of RRP exocytosis in IHCs} during repetitive stimulation

Analysis of trial-to-trial fluctuations requires the $\Delta C_{\mathrm{m}}$ responses to be stationary enough to collect a sufficiently large ensemble of $\Delta C_{\mathrm{m}}$ values while the release rate $\lambda$ remains virtually constant. To avoid rapid rundown of the exocytic IHC response we used the perforated-patch configuration and kept an interstimulus interval of $\geq 12$ s (sufficient for $>90 \%$ RRP recovery in mature IHCs) (Moser and Beutner, 2000). We observed a whole spectrum of secretory behaviors during repetitive release of the RRP (Fig. 4) and the reasons behind these variable time courses remain unknown.

Approximately 3 of 15 mature and 5 of 14 immature IHCs showed a pronounced initial rundown of RRP exocytosis. The evoked $\Delta C_{\mathrm{m}}$ finally approached the noise floor defined by the spontaneous $\Delta C_{\mathrm{m}}$ (Fig. 4A), despite the fact that the $\mathrm{Ca}^{2+}$ current was not substantially reduced over the time of the exocytosis rundown (data not shown). This loss of depolarizationinduced $C_{\mathrm{m}}$ increments argues strongly against the presence of a nonexocytic $\Delta C_{\mathrm{m}}$ as observed in cells with high expression levels of voltage-gated sodium channels (Horrigan and Bookman, 1994; Moser and Neher, 1997b) or ion transporter molecules (Santos-Sacchi, 1991). We would expect these depolarization-evoked movements of gating charges in the bilayer to be much more stable over time than exocytosis (Santos-Sacchi, 1991; Horrigan and Bookman, 1994; Moser and Neher, 1997a). The mechanism of the exocytic rundown is at present unknown. We assume it is mostly caused by fatigued resupply of readily releasable vesicles. A slow decline resulting in a nearly stationary $\Delta C_{\mathrm{m}}$ was found in $\sim 5$ of 15 mature and 5 of 14 immature IHCs (Fig. 4C). Finally, 7 of 15 mature and 4 of 14 immature IHCs showed an intermediate pattern (Fig. $4 B$ ).

\section{Analysis of trial-to-trial}

\section{$\Delta C_{\mathrm{m}}$ fluctuations}

The analysis is illustrated step-by-step in Figure 5 for a representative experiment in a mature IHC. The top panel of Figure $5 A$ shows the original evoked $\Delta C_{\mathrm{m}}$ (filled circles) and spontaneous $\Delta C_{\mathrm{m}}$ (empty circles, preceding the depolarization). To avoid contaminating variance contributions resulting from the nonstationarity of release, we then removed the trend of the $\Delta C_{\mathrm{m}}$ data by subtracting from the data a (1) forward-reverse low-pass filtered version of the data (Fig. 5A, gray line) (see Materials and Methods for details) or (2) double-exponential fit (to evoked $\Delta C_{\mathrm{m}}$, and a linear fit for spontaneous $\Delta C_{\mathrm{m}}$ ).

These trend-corrected data (Fig. $5 A$, correction 1, second plot) were used to calculate the ensemble variance. Spontaneous
$\Delta C_{\mathrm{m}}$ were treated accordingly. One expects trend correction 1 to remove some trial-to-trial variance and, therefore, to result in an underestimate of $C_{\text {app }}$. We assessed the direction and size of such bias in surrogate $C_{\mathrm{m}}$ data simulating a spectrum of secretory behaviors similar to what we observed experimentally (using a fixed $\mu$ and exponentially declining $\lambda$ ) (see Materials and Methods). We found a marginal bias of $<5 \%$ toward smaller $C_{\text {app }}$ estimates. Trend correction 2 is expected to add some artificial variance, because the data often deviated from a truly double exponential decay. Hence, the two types of trend correction resulted in lower and upper bounds for the true $C_{\text {app }}$ estimate ( $\mathrm{Ta}-$ 

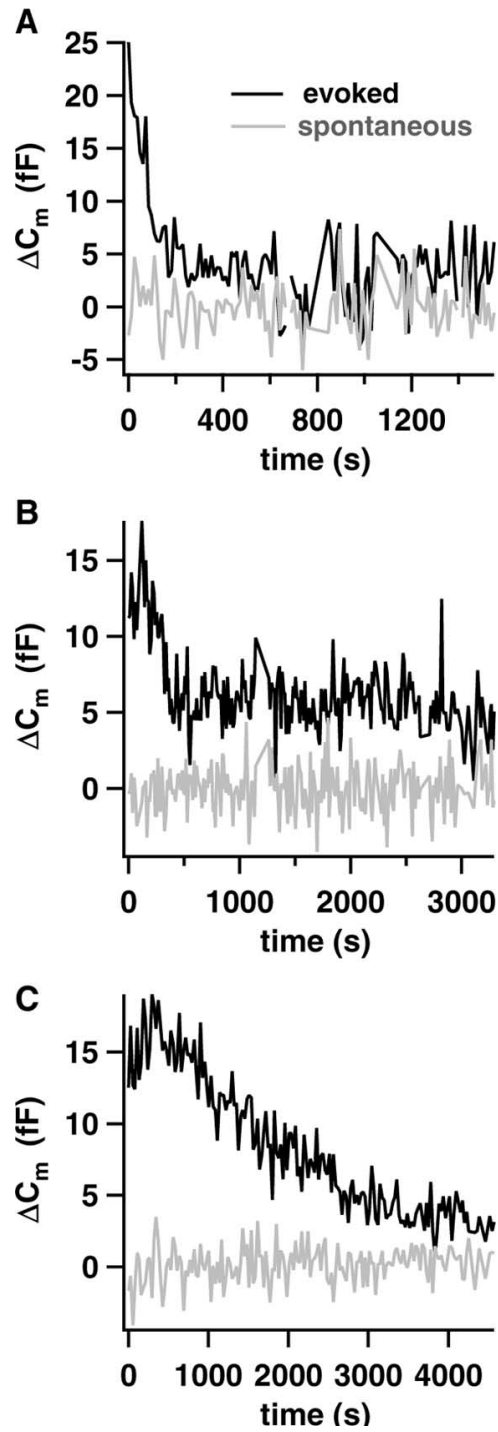

Figure 4. Rundown of RRP exocytosis in IHCs during repetitive stimulation. A-C, Time courses of evoked and spontaneous $\Delta C_{\mathrm{m}}$ in representative IHCs featuring varying degrees of exocytic rundown. $\mathrm{A} \mathrm{K}^{+}$-gluconate-based pipette solution $\left(I_{1}\right)$ was used for the experiments shown in Figure 4

ble 2). Because of the observed rundown we generally used subsequent ensembles of only five $\Delta C_{\mathrm{m}}$ estimates (indicated by gray column) to obtain variance (from residuals) and mean (from raw $\Delta C_{\mathrm{m}}$ data) estimates. Each analysis bin was then shifted without overlap. We calculated five individual series of ensemble estimates each starting shifted by one $\Delta C_{\mathrm{m}}$. Ensemble means and variances for evoked and spontaneous $\Delta C_{\mathrm{m}}$ data are shown as a function of time in the lowest two panels of Figure $5 \mathrm{~A}$.

The correlation of ensemble variances and means of the representative experiment is demonstrated in the scatterplot in Figure $5 B$, which pools the estimates of all bins analyzed (five series of nonoverlapping bins after correction 1 in different grays, filled circles: evoked $\Delta C_{\mathrm{m}}$, open circles: dummy $\left.\Delta C_{\mathrm{m}}\right) . C_{\text {app }}$ was estimated as the slope of the linear regression between variances and means independently for each series (corresponding grays) including the dummy $\Delta C_{\mathrm{m}}$. Inclusion of the spontaneous $\Delta C_{\mathrm{m}}$ was justified by the lack of a nonexocytic $\Delta C_{\mathrm{m}}$ and improved the certainty of the $C_{\text {app }}$ estimation.

Estimation of confidence intervals for the linear regression of variance and mean is not straight forward because of error in the
A
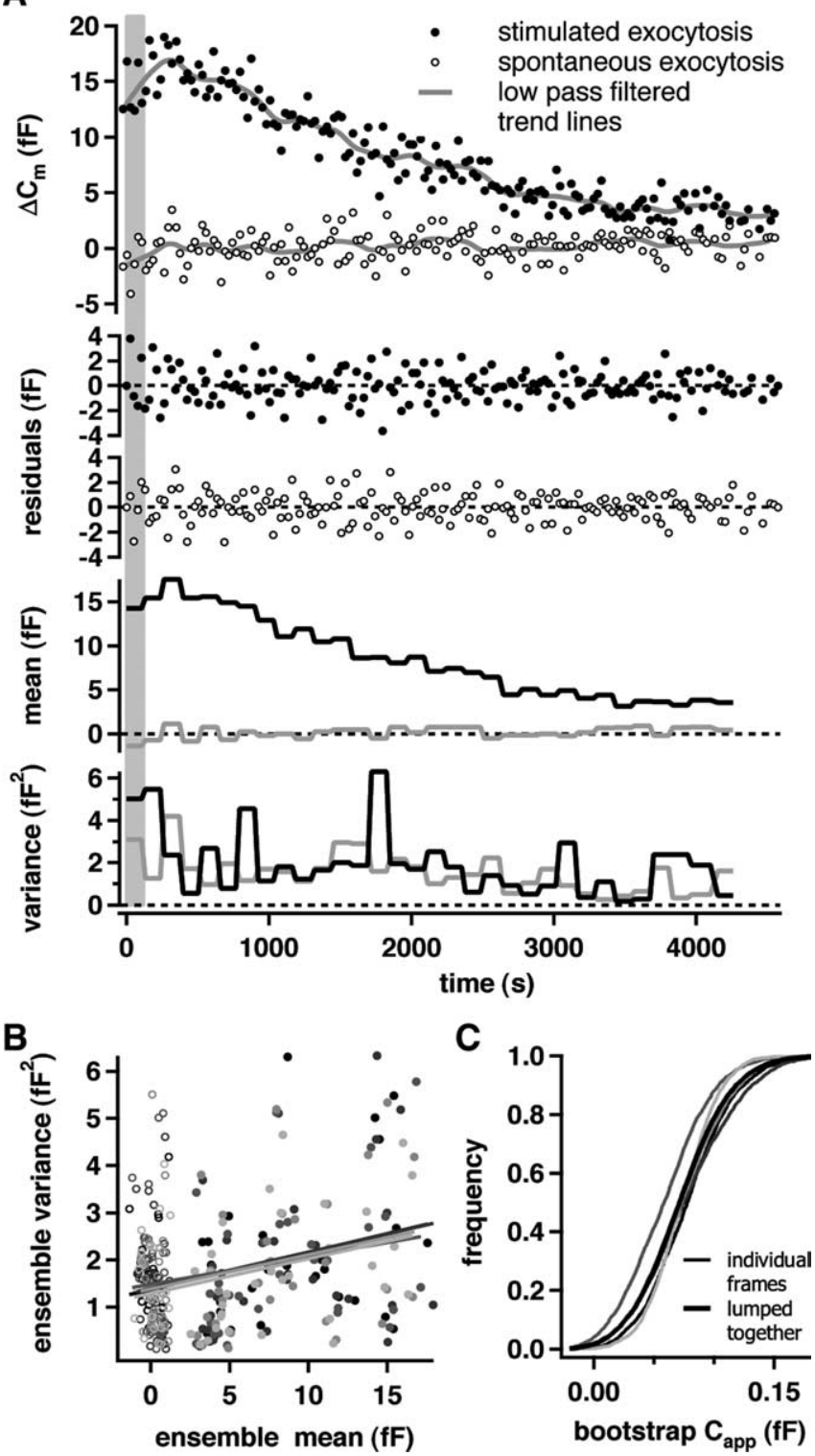

Figure 5. Analysis of the fusion event size by trial-to-trial fluctuations in membrane capacitance increases. Summary of the analysis in one representative mature IHC. $A$, top, evoked $\Delta C_{m}$ (filled symbols, depolarizations to peak $\mathrm{Ca}^{2+}$ current potential) show exocytosis that declines over time. Dummy stimuli do not lead to exocytosis, but spontaneous $\Delta C_{m}$ (empty circles) reflecting the measurement noise. Lines on top of the markers represent trend lines obtained from low-pass filtering of the $\Delta C_{\mathrm{m}}$ values and were used to remove any trend from the data (residuals, second plot) to calculate the variance. Ensemble estimates for the mean $\Delta C_{m}$ (third panel) and its variance (fourth panel, variance of the residuals) were calculated in nonoverlapping windows of five $\Delta C_{m}$ values each (first window depicted in gray). This was done in five series, each starting shifted by one point. $\boldsymbol{B}$, Scatterplot of the variance and means obtained in the five series of ensembles (each shade represents one of the five series). Lines are results of linear regression between variances and means of the five series considering evoked (filled symbols) and spontaneous (empty symbols) $\Delta C_{m}$ values. The slope of a line represents the apparent size of elementary exocytic events $\left(C_{\text {app }}\right)$. $C$, Bootstrapping was used to estimate the error of the cell's mean $C_{\text {app }}$. Each gray line represents the cumulative distribution of $C_{\text {app }}$ from 500 bootstrap samples of mean/variance data from $(\boldsymbol{B})$ for one of the five series (same color code as in $\boldsymbol{B}$ ). These five distributions were lumped together for generation of this cell's $\boldsymbol{C}_{\text {app }}$ distribution (black line; mean, $72 \mathrm{aF} ; 95 \% \mathrm{Cl} ; 4,146 \mathrm{aF}$ ). $\mathrm{AK}^{+}$-gluconate-based pipette solution $\left(I_{1}\right)$ was used for the experiment shown in Figure 5.

estimates of the mean (measurement error) and heteroscedasticity of the variance estimates (error varying with the mean). Therefore, we calculated CIs by a nonparametric randomization 
Table 2. Results of nonstationary fluctuation analysis of IHC $\Delta C_{m}$

\begin{tabular}{|c|c|c|c|c|c|c|}
\hline & $C_{\mathrm{sv}}(\mathrm{aF}) \pm \mathrm{SEM}$ & $C_{\text {app, weight }}(\mathrm{aF})[95 \% \mathrm{Cl}]$ & $\mu_{\text {weight }}[95 \% \mathrm{Cl}]$ & $C_{\text {app }}(\mathrm{aF})[95 \% \mathrm{Cl}]$ & $\mu[95 \% \mathrm{Cl}]$ & Trend corr. \\
\hline \multirow[t]{2}{*}{ Mature, $\mathrm{K}^{+}(n=15)$} & \multirow[t]{2}{*}{$45 \pm 1(n=574)$} & $95[55,141]$ & $1.6[1.1,2.1]$ & $94[28,159]$ & $1.6[0.8,2.3]$ & 1 \\
\hline & & $102[58,149]$ & $1.6[1.1,2.2]$ & $98[25,174]$ & $1.6[0.8,2.4]$ & 2 \\
\hline Mature $\mathrm{K}^{+}$, ryanodine, $(n=4)$ & \multirow{3}{*}{$48 \pm 1(n=602)$} & $145[72,221]$ & $2.1[1.3,3.0]$ & $160[72,251]$ & $2.3[1.3,3.3]$ & 1 \\
\hline \multirow[t]{2}{*}{ Immature $\mathrm{K}^{+}(n=14)$} & & $147[96,200]$ & $2.0[1.5,2.6]$ & $155[87,223]$ & $2.1[1.4,2.8]$ & 1 \\
\hline & & $166[117,223]$ & $2.2[1.7,2.8]$ & $170[110,240]$ & $2.3[1.7,3.0]$ & 2 \\
\hline Immature $\mathrm{Cs}^{+}(n=6)$ & & $133[63,208]$ & $1.9[1.2,2.7]$ & $132[41,232]$ & $1.9[1,2.9]$ & 1 \\
\hline
\end{tabular}

Summary of the results obtained with and without weighting as well as with the two different trend corrections. Trend correction 1 corresponds to subtraction of a trend function obtained by low-pass filtering of the data, and correction 2 used subtraction of a double-exponential fit. The average number of synaptic vesicles involved in a fusion event $(\mu)$, was calculated according to $\mu=\left(C_{\text {app }} / C_{\text {sv }}+1\right) / 2$. corr., $C_{\text {orrection. }}$.

method (bootstrapping) (for details, see Materials and Methods) (Davison and Hinkley, 2003). Figure 5C shows the cumulative density functions (CDFs) of the five bootstrap distributions and the resulting mean $C_{\text {app }}$ distribution. For this particular cell we obtained a mean $C_{\mathrm{app}}$ of 72 $\mathrm{aF}(95 \% \mathrm{CI} ; 4,146 \mathrm{aF})$ after correction 1 (subtraction of a low pass filtered version) and $75 \mathrm{aF}$ (95\% CI; 6, $146 \mathrm{aF}$ ) after correction 2 (subtraction of a double exponential fit). Jackknife (see Materials and Methods) (Davison and Hinkley, 2003) was used to identify outliers whose removal reduced the CI of the slope estimator by $>20 \%$. We rejected only one or two $\Delta C_{\mathrm{m}}$ estimates in 13 of 29 IHCs whereas in 13 IHC we did not exclude any $\Delta C_{\mathrm{m}}$ (Fig. 5 , experiment).

Cell-population averages for $C_{\text {app }}$

The bootstrap distributions for $C_{\text {app }}$ varied in mean and width among the IHCs with some IHCs showing CDFs spanning tens of attoFarads whereas CDFs of others covered hundreds of attoFarads (Fig. 6). These different confidence intervals might in principle reflect different properties of the unknown, underlying size distribution of elementary release events (Fig. $1 A, B$ ). However, we found that narrow confidence intervals typically coincided with large average $\Delta C_{\mathrm{m}}$, high number of $\Delta C_{\mathrm{m}}$ trials and low variance of spontaneous $\Delta C_{\mathrm{m}}$, suggesting that the observed CI heterogeneity rather reflects recording quality and the number of independent data points obtained for a cell. The influence of these factors is quantified by the expression $\frac{\sigma^{2}\left(\Delta C_{m}^{\text {dummy }}\right)}{\sqrt{N_{\Delta C_{m}}}\left\langle\Delta C_{m}^{\text {stimulus }}\right\rangle}$ that can be obtained directly from the raw data: number of sweeps $N_{\Delta C_{m}}$, average size of the depolarization evoked capacitance jump $\left\langle\Delta C_{m}^{\text {stimulus }}\right\rangle$ and the variance of the spontaneous capacitance fluctuations $\sigma^{2}\left(\Delta C_{m}^{\text {dummy }}\right)$. Simulating $C_{\mathrm{m}}$ data, so that all experiments used the same release model $(\mu, \lambda)$, we found that the above factor is in fact a good predictor of CI size $\left(r_{\text {Pearson }}=0.8 ; 64 \%\right.$ explained variance $)$. Data from all cells in a class (same age group, solutions and stimulus) were pooled in two ways: with or without weighting the individual observations
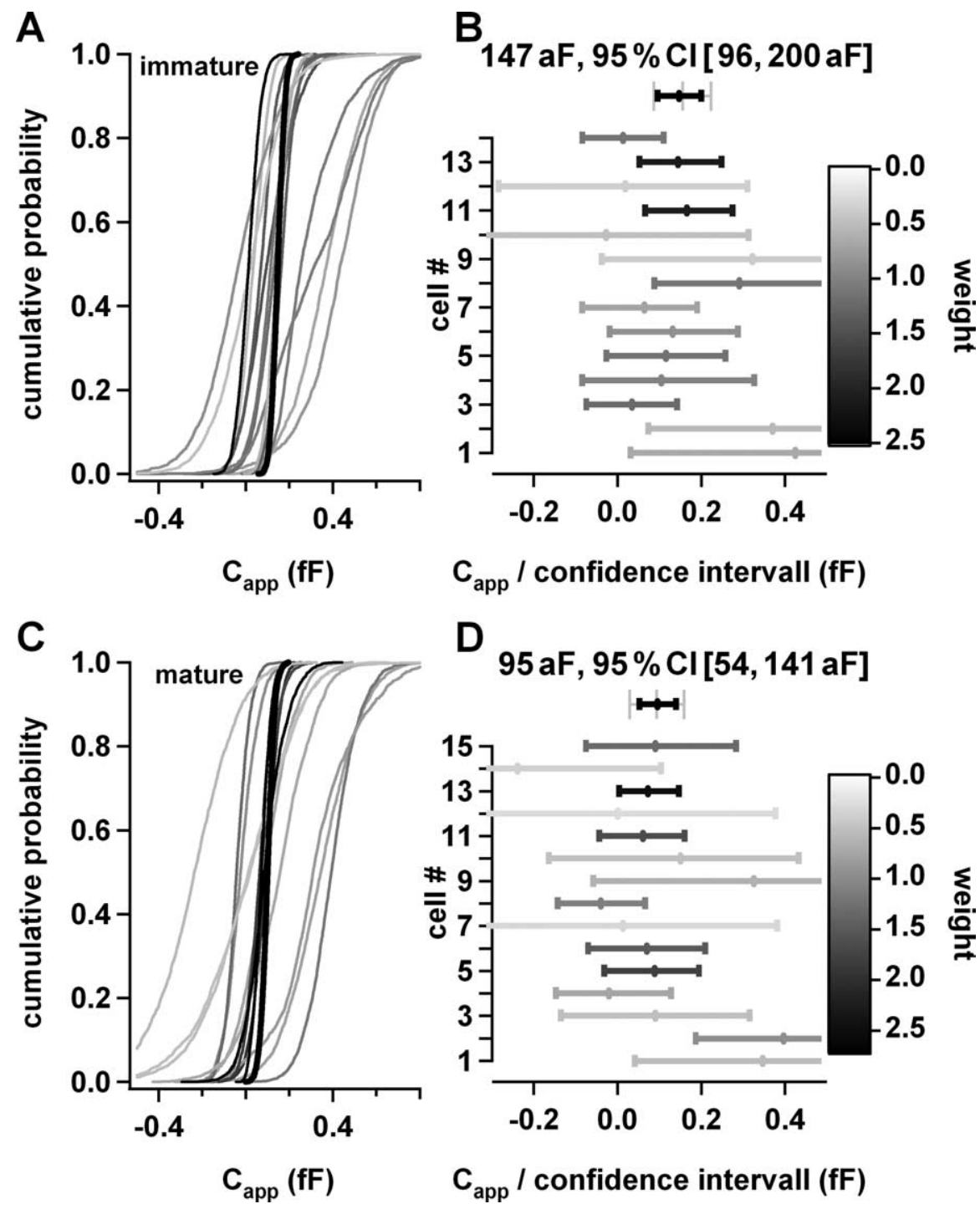

Figure 6. Obtaining population averages for $C_{\text {app }}$. $A$ and $\boldsymbol{C}$ display the $C D F s$ of the bootstrap distributions of $C_{\text {app }}$ for the individual immature $(n=14)$ and mature $(n=15)$ IHCs and their average CDFs (thick, black trace). The different colors code the weight of the cells (see Results) (grayscale map). B, $C_{\text {app }}$ means and their $95 \%$ confidence intervals for individual immature IHCs. The uppermost black bar in $\boldsymbol{B}$ represents the mean and the confidence interval for a weight average of the individual cells. The underlying gray bar depicts the result of nonweighted averaging. $\boldsymbol{D}$, same as $\boldsymbol{B}$ but for mature $\mathrm{HHCs}$. $\mathrm{KK}^{+}$-gluconate-based pipette solution $\left(I_{1}\right)$ was used for the experiments shown in Figure 6.

by $\frac{\sqrt{N_{\Delta C_{m}}}\left\langle\Delta C_{m}^{\text {stimulus }}\right\rangle}{\sigma^{2}\left(\Delta C_{m}^{\text {dummy }}\right)}$. As expected, weighting resulted in slightly narrower confidence intervals for the population averages of $C_{\mathrm{app}}$ than nonweighted pooling in both immature and mature IHCs.

Table 2 summarizes the $C_{\text {app }}$ estimates and their CIs. The CIs of mature (55-149 aF) and immature (96-223 aF) IHCs overlap, 
but there is a tendency toward larger elementary release events at immature synapses. For both groups $C_{\text {app }}$ was significantly larger than $C_{\mathrm{sv}}$, indicating coordination of release.

\section{Testing the impact of $\mathrm{Ca}^{2+}$ induced $\mathrm{Ca}^{2+}$ release on $C_{\text {app }}$} CICR was shown to trigger multivesicular release at a CNS synapse (Llano et al., 2000). CICR has been reported to contribute a fast component of the submembrane $\mathrm{Ca}^{2+}$ rise in IHCs (Kennedy and Meech, 2002) and, hence, could potentially serve as a signal coordinating the release of multiple vesicles at the IHC active zone. Here, we explored this possibility by testing the effects of ryanodine and intracellular $\mathrm{Cs}^{+}$, both shown to block the fast component of the submembrane $\mathrm{Ca}^{2+}$ rise in IHCs (Kennedy and Meech, 2002) associated with CICR. We did not find a significantly smaller mean $\mathrm{C}_{\mathrm{app}}$ in four mature IHCs when they had been incubated with $100 \mu \mathrm{M}$ ryanodine in the extracellular solution (Table 2). Likewise, intracellular $\mathrm{Cs}^{+}(150 \mathrm{~mm}) \mathrm{did}$ not result in a significantly smaller mean $C_{\text {app }}$ in six immature IHCs (Table 2). Therefore, coordination of vesicle release in IHCs seems not to involve CICR.

\section{Discussion}

This study addressed the mechanism of exocytosis at the hair cell ribbon synapse before and after the onset of hearing using presynaptic measurements. We estimated the mean apparent size of an elementary fusion event $\left(C_{\text {app }}\right)$ by analysis of nonstationary fluctuations of exocytic $\Delta C_{\mathrm{m}}$. Comparison with a morphological estimate of single vesicle capacitance $C_{\mathrm{sv}}$ demonstrates evidence for coordinated vesicle fusion. The fluctuation analysis extends the use of whole-cell $C_{\mathrm{m}}$ measurements for studying hair cell presynaptic function to the microscopic properties of exocytosis despite the unfavorable signal to background noise ratio. The compound Poisson model explored here offers a framework also for future analysis and simulations of exocytosis at ribbon synapses. It will as well be useful for describing other physiological stochastic processes with coordinated elementary events such as coupled gating of ion channels (Lehnart et al., 2003; Molina et al., 2006).

The results indicate that coordinated exocytosis governs a substantial fraction of release events at mouse hair cell ribbon synapses. In immature IHCs about half the release events involve coordinated fusion. Our estimate of the average number of vesicles per fusion event (two) is half of that derived from EPSC distributions of immature rat synapses (Glowatzki and Fuchs, 2002). Coordinated release at mature and immature IHC synapses probably does not involve CICR.

\section{Capacitance fluctuation analysis reports coordinated fusion of vesicles in IHCs}

The mechanism of exocytosis at the hair cell ribbon synapse remains an interesting but unsolved issue. Previously, postsynaptic recordings from immature rat afferent postsynaptic boutons (Glowatzki and Fuchs, 2002) and frog papillar afferent nerve fibers (Keen and Hudspeth, 2006) demonstrated highly synchronized vesicle release and suggested presynaptic coordination of exocytosis. Although this is likely the case, it was not clear how much of the synchronization reflects coordination of release at individual active zones. This is because some of these postsynaptic currents might have resulted from release at more than one active zone (Sobkowicz et al., 1982; Keen and Hudspeth, 2006) and some additional variation of EPSC size may have occurred from variable transmitter filling of vesicles (Wu et al., 2007) and/or the distribution of postsynaptic transmitter receptors. One advantage of the whole-cell capacitance measurements is avoiding complications inherent with the postsynaptic detection of transmitter release. Despite the unfavorable signal to background noise ratio, our fluctuation analysis determined the apparent sizes of elementary exocytic events in IHCs with good accuracy and supports the statistical dependence among the release of individual vesicles.

Figure 7 interprets our estimates of $C_{\text {app }}$, the apparent size of the elementary fusion event in the framework of the compound Poisson release model as introduced in this work. Assuming that the size distribution of fusion events can be described by geometric distributions (see above) $\sim 65 \%$ of the fusion events in mature IHCs involve one vesicle and most of the remaining events involve coordinated release of only two or three synaptic vesicles. Events with larger numbers of synaptic vesicles can occur, but are much less likely. Interestingly, we observed a trend toward larger average release events in mouse IHCs at an earlier developmental stage. As our morphological vesicle capacitance estimates were only marginally different this may argue for more coordination of release at immature synapses. Alternatively, mature and immature synaptic vesicles may differ in their protein complement, which could result in different specific membrane capacitances. Our estimate for the coordination of exocytosis in immature cells is only half as big as estimated from rat postsynaptic recordings by Glowatzki and Fuchs (2002). Hence, both postsynaptic and presynaptic measurements of transmitter release indicate significant coordination of synaptic vesicle exocytosis at immature IHCs active zones but report different degrees of coordination.

\section{Limitations of the presented analysis}

$C_{\mathrm{m}}$ recordings measure membrane surface changes caused by exocytosis and endocytosis, thus, in principle, endocytosis could affect our $C_{\text {app }}$ estimate. To date, two endocytic modes have been reported in IHCs: $(1)$ rapid endocytosis $(\tau \sim 0.3 \mathrm{~s})$ (Beutner et al., $2001)$, only present after large global $\left[\mathrm{Ca}^{2+}\right]_{\mathrm{i}}$ rises evoked by $\mathrm{Ca}^{2+}$ uncaging, and (2) slow compensatory endocytosis $(\tau \sim 7 \mathrm{~s})$ (Moser and Beutner, 2000) that also follows exocytosis evoked by voltage-gated $\mathrm{Ca}^{2+}$ influx. The average $C_{\mathrm{m}}$ traces of mature and immature IHCs did not show a major additional decline after the depolarization (Fig. $3 B, C$ ) and the variance among spontaneous $\Delta C_{\mathrm{m}}$ before and after the depolarization was not different (data not shown). Therefore, we exclude major effects of compensatory endocytosis on our $C_{\text {app }}$ estimate. A $C_{\mathrm{m}}$ transient $(\tau \sim 0.03 \mathrm{~s})$ that was much faster than rapid endocytosis in hair cells (Beutner et al., 2001) or retinal bipolar nerve terminals ( $\sim 1 \mathrm{~s})$ (von Gersdorff and Matthews, 1994; Neves et al., 2001) occurs after repolarization (Figs. $3 \mathrm{~A}, \mathrm{C}$ ). It most likely reflects a bleed through of a membrane conductance change and was excluded from this analysis by discarding the first $40 \mathrm{~ms}$. Although unlikely, we cannot ultimately exclude the existence of a small component of exocytosis that involved more coordination, but went undetected because the fused membrane got retrieved with a similar ultrafast kinetics.

Errors in the morphological approximation of $C_{\mathrm{sv}}$ (e.g., caused by use of an inappropriate specific membrane capacitance or vesicle shrinkage during chemical fixation), could lead to erroneous conclusions on the coordination of vesicle release. Estimates of specific capacitance from a variety of cellular membranes range from $5 \mathrm{fF} \mu \mathrm{m}^{-2}$ (Solsona et al., 1998) to $30 \mathrm{fF} \mu \mathrm{m}^{-2}$ (Graf et al., 1995). Potential reasons for this variation include 
biological differences (e.g., membrane protein content and composition) and methodological differences. For the purpose of the present study, we used a specific membrane capacitance of 10 $\mathrm{fF} \mu \mathrm{m}^{-2}$, close to what was obtained for the giant secretory vesicles of beige mouse mast cells (11.7 $\mathrm{fF} \mu \mathrm{m}^{-2}$ ) (Breckenridge and Almers, 1987), the value of $8 \mathrm{fF} \mu \mathrm{m}^{-2}$ derived from the capacitance of mouse chromaffin granules (1.3 fF) (Moser and Neher, 1997) and their surface (calculated from the mean diameter of $227 \mathrm{~nm}$ ) (Grabner et al., 2005) and the average value found for the plasma membrane in several neurons using nucleated patches ( $\sim 9 \mathrm{fF} \mu \mathrm{m}^{-2}$ ) (Gentet et al., 2000).

To test whether shrinkage compromised our measurements we compared our diameter estimates to those of frozen and of chemically fixed CNS synaptic vesicles observed by the same experimenter (Fig. 2C) (data not shown). We found the vesicle diameter distributions of mature IHCs and both samples of CNS synaptic vesicles to overlap arguing against substantial shrinkage in our present study. Our average $C_{\mathrm{sv}}$ estimates of $48 \mathrm{aF}$ (immature) and $45 \mathrm{aF}$ (mature) rested between previous morphological estimates [e.g., 26 aF (von Gersdorff et al., 1996); $29 \mathrm{aF}$ (Khimich et al., 2005); 37 aF (Lenzi et al., 1999) ] and functional estimates for synaptic vesicles [calyx of Held, $66 \mathrm{aF}$ (Wu et al., 2007); $80 \mathrm{aF}$ (He et al., 2006); $80 \mathrm{aF}$ (Sakaba, 2006)] and synaptic-like microvesicles (50 aF) (Klyachko and Jackson, 2002). We conclude that we had previously underestimated $C_{\text {sv }}$ in IHCs $(29 \mathrm{aF})$ (Khimich et al., 2005) most likely attributable to shrinkage. Future direct recordings of vesicle fusion events by cell-attached capacitance measurements will provide a reliable estimate for interpretation of wholecell $C_{\mathrm{m}}$ rises as rates of synaptic vesicle release.

Because of the exponential-like EPSC amplitude distributions (Glowatzki and Fuchs, 2002; Keen and Hudspeth, 2006) our study focused on geometric statistics for the inner process, but other statistics may also be applicable (Furukawa et al., 1982). Our assumption of a Poisson point process was motivated by the exponential interevent interval distribution observed during recordings of postsynaptic currents (Glowatzki and Fuchs, 2002) and the rapid resupply of readily releasable vesicles that counteracts the consumption of the RRP during exocytosis (Moser and Beutner, 2000; Edmonds et al., 2004; Spassova et al., 2004). If the RRP was widely consumed by our 10 or $20 \mathrm{~ms}$ stimuli, we might have underestimated the degree of coordination of vesicle release. We used an elevated $\left[\mathrm{Ca}^{2+}\right]_{\mathrm{e}}$ to augment $\mathrm{Ca}^{2+}$ currents and exocytosis in these long-lasting experiments because, unfortunately, the average size of the evoked capacitance jumps evoked at physiological $\left[\mathrm{Ca}^{2+}\right]_{\mathrm{e}}(1.3 \mathrm{~mm})$ was too low to estimate $C_{\text {app }}$ with sufficient certainty. The stronger presynaptic $\left[\mathrm{Ca}^{2+}\right]$ signal would probably favor rather than inhibit coordination of release (e.g., by promoting homotypic fusion of vesicles).

\section{Mechanisms underlying coordination of vesicle release at a ribbon synapse}

Mechanisms potentially underlying coordination of vesicle release include (1) synchronization of individual vesicle release events triggered by a common $\mathrm{Ca}^{2+}$ signal (2) homotypic vesicle to vesicle fusion before exocytosis (compound exocytosis) (Scepek and Lindau, 1993; Lollike et al., 2002) or (3) cumulative homotypic fusion of vesicles to each other during exocytosis (Rohlich et al., 1971; Takahashi et al., 2004). Our fluctuation analysis cannot distinguish among these possibilities, but argues against an essential role of CICR in coordination of release in IHCs. Future experiments including cell-attached capacitance measurements of individual presynaptic fusion events and electron microscopy of stimulated synapses are required to further address the mechanism coordinating release at the hair cell ribbon synapse. 


\section{Appendix}

\section{Derivation of the mean and variance of a general compound process}

We consider two coupled stochastic variables $K$ and $R$ that are integer and non-negative. During each realization of the combined process, $k_{j}$ gives the number of events $r_{i}$ occurring. Let the respective probability distributions be $Q\left(K=k_{j}\right)$ and $\left(r=r_{i}\right)$, then the sum $S$ of all $r_{i}$ that occurred in one realization is as follows:

$$
s=\sum_{i=1}^{k} r_{i}
$$

In the following, we derive the expectation value and the variance of the stochastic variable $S$ under the assumption that all $r_{i}$ are independent. An overline denotes averaging over release events $R$, whereas brackets denote averaging over numbers $K$ of release events. In this notation the expectation value of $K$ is $\langle K\rangle$ and the respective variance is $\sigma_{K}^{2}$. Accordingly $P^{\prime}(R)$ has the expectation value $\bar{R}$ and the variance $\sigma_{K}^{2}$.

For a fixed $k$, the distribution $\operatorname{Pr}\left(S_{k}=s\right)$ has a conditional $(K=k)$ mean

$$
\bar{S}_{k}=\sum_{r_{1}, r_{2}, r_{k} i=1} \sum_{i}^{k} r_{i} P\left(r_{1}, r_{2}, \ldots r_{k}\right)
$$

Because the events $r_{i}$ are independent, we can use the marginal distribution

$$
P^{\prime}\left(r_{i}\right)=\sum_{r_{2}, r_{3}, \ldots r_{k}} P\left(r_{1}, r_{2}, \ldots r_{k}\right)
$$

to factorize the probability $P$ :

$$
P\left(r_{1}, r_{2}, \ldots r_{k}\right)=\prod_{i} P^{\prime}\left(r_{i}\right) .
$$

This allows to simplify Equation A2:

$$
\begin{gathered}
\bar{S}_{k}=\sum_{r_{1}}\left[r_{1} \sum_{r_{2}, \ldots r_{k}} P\left(r_{1}, r_{2}, \ldots r_{k}\right)+\sum_{r_{2}} r_{2} \sum_{r_{3}, \ldots r_{k}} P\left(r_{1}, r_{2}, \ldots r_{k}\right)\right. \\
+\sum_{r_{3} r_{3} \sum_{r_{2}, r_{4} \ldots r_{k}} P\left(r_{1}, r_{2}, \ldots . r_{k}\right)+\ldots}+\sum_{\left.r_{k} r_{k} \sum_{r_{2}, \ldots r_{k-1}} P\left(r_{1}, r_{2}, \ldots r_{k}\right)\right]} \\
\left.\bar{S}_{k}=\sum_{r_{1}\left[r_{1} P^{\prime}\left(r_{1}\right)+\sum_{r_{2}} r_{2} P^{\prime \prime}\left(r_{1}, r_{2}\right)+\sum_{r_{3}} r_{3} P^{\prime \prime}\left(r_{1}, r_{3}\right)+\ldots\right.}+\sum_{r_{k}} r_{k} P^{\prime \prime}\left(r_{1}, r_{k}\right)\right] \\
\bar{S}_{k}=\sum_{r_{1} r_{1} P^{\prime}\left(r_{1}\right)+}+\sum_{r_{2}} r_{2} P^{\prime}\left(r_{2}\right)+\sum_{r_{3}} r_{3} P^{\prime}\left(r_{3}\right)+\ldots \\
+\sum_{r_{k}} r_{k} P^{\prime}\left(r_{k}\right)
\end{gathered}
$$

$$
\bar{S}_{k}=\sum_{i=1}^{k} \sum_{r_{1}} r_{i} P^{\prime}\left(r_{i}\right)=k \sum_{r_{i}} r_{i} P^{\prime}\left(r_{i}\right) .
$$

The last sum represents $\bar{R}$. Hence, the mean of $S$ is given by the product of the means of the inner and the outer process:

$$
\langle\bar{S}\rangle=\langle K\rangle \cdot \bar{R}
$$

The conditional $(K=k)$ variance of $S$ is

$$
\overline{\sigma_{S_{k}}^{2}}=k \sigma_{R}^{2}=k \sum_{r_{i}}\left(r_{i}-\mu\right)^{2} P^{\prime}\left(r_{i}\right) .
$$

So, the total variance of $S$ is then

$$
\sigma_{S}^{2}=\left\langle\overline{\left(s_{k}-\langle\bar{S}\rangle\right)^{2}}\right\rangle .
$$

Resolving the square and the averages this can be simplified:

$$
\begin{aligned}
\sigma_{S}^{2} & =\left\langle\overline{s_{k}^{2}}\right\rangle+\langle\bar{S}\rangle^{2}-2\left\langle\overline{s_{k}}\langle\bar{S}\rangle\right\rangle \\
\sigma_{S}^{2} & =\left\langle\overline{s_{k}^{2}}\right\rangle-\langle\bar{S}\rangle^{2}
\end{aligned}
$$

Using the definition of variance $\bar{\sigma}_{S_{k}}^{2}=\bar{S}_{k}^{2}-\bar{S}_{k}^{2}$ and the equations A6 and A7, we can substitute the first bracket to reduce equation A9 to known factors.

$$
\begin{aligned}
\sigma_{S}^{2} & =\left\langle\overline{\sigma_{S_{k}}^{2}}\right\rangle+\left\langle{\overline{s_{k}}}^{2}\right\rangle-\bar{R}^{2}\langle K\rangle^{2} \\
\sigma_{S}^{2} & =\left\langle k \sigma_{R}^{2}\right\rangle+\left\langle k^{2} \bar{R}^{2}\right\rangle-\bar{R}^{2}\langle K\rangle^{2} \\
\sigma_{S}^{2} & =\langle k\rangle \sigma_{R}^{2}+\bar{R}^{2}\left(\left\langle k^{2}\right\rangle-\langle K\rangle^{2}\right) \\
\sigma_{S}^{2} & =\langle K\rangle \sigma_{R}^{2}+\bar{R}^{2} \sigma_{K}^{2}
\end{aligned}
$$

Equations A6 and A10 are the general forms of Equations 2 and 3. They can be combined to obtain the relation:

$$
\frac{\sigma_{S}^{2}}{\langle\bar{S}\rangle}=\frac{\sigma_{R}^{2}}{\bar{R}}+\bar{R} \cdot \frac{\sigma_{k}^{2}}{\langle K\rangle} .
$$

\section{References}

Auger C, Kondo S, Marty A (1998) Multivesicular release at single functional synaptic sites in cerebellar stellate and basket cells. J Neurosci 18:4532-4547.

Beutner D, Voets T, Neher E, Moser T (2001) Calcium dependence of exocytosis and endocytosis at the cochlear inner hair cell afferent synapse. Neuron 29:681-690.

Brandt A, Striessnig J, Moser T (2003) CaV1.3 channels are essential for development and presynaptic activity of cochlear inner hair cells. J Neurosci 23:10832-10840.

Breckenridge LJ, Almers W (1987) Final steps in exocytosis observed in a cell with giant secretory granules. Proc Natl Acad Sci USA 84:1945-1949.

Davison AC, Hinkley DV (2003) Bootstrap methods and their application, Ed 5. Cambridge, UK: Cambridge UP.

Del Castillo J, Katz B (1954) Quantal components of the end-plate potential. J Physiol (Lond) 124:560-573.

Edmonds BW, Gregory FD, Schweizer FE (2004) Evidence that fast exocytosis can be predominantly mediated by vesicles not docked at active zones in frog saccular hair cells. J Physiol (Lond) 560:439-450.

Fuchs PA, Glowatzki E, Moser T (2003) The afferent synapse of cochlear hair cells. Curr Opin Neurobiol 13:452-458.

Furukawa T, Kuno M, Matsuura S (1982) Quantal analysis of a decremental response at hair cell-afferent fibre synapses in the goldfish sacculus. J Physiol 322:181-195.

Gentet LJ, Stuart GJ, Clements JD (2000) Direct measurement of specific membrane capacitance in neurons. Biophys J 79:314-320.

Glowatzki E, Fuchs PA (2002) Transmitter release at the hair cell ribbon synapse. Nat Neurosci 5:147-154.

Grabner CP, Price SD, Lysakowski A, Fox AP (2005) Mouse chromaffin cells have two populations of dense core vesicles. J Neurophysiol 94:20932104.

Graf J, Rupnik M, Zupancic G, Zorec R (1995) Osmotic swelling of hepatocytes increases membrane conductance but not membrane capacitance. Biophys J 68:1359-1363. 
He L, Wu XS, Mohan R, Wu LG (2006) Two modes of fusion pore opening revealed by cell-attached recordings at a synapse. Nature 444:102-105.

Horrigan FT, Bookman RJ (1994) Releasable pools and the kinetics of exocytosis in adrenal chromaffin cells. Neuron 13:1119-1129.

Keen EC, Hudspeth AJ (2006) Transfer characteristics of the hair cell's afferent synapse. Proc Natl Acad Sci USA 103:5537-5542.

Kennedy HJ, Meech RW (2002) Fast $\mathrm{Ca}^{2+}$ signals at the mouse inner hair cell synapse: a role for $\mathrm{Ca}^{2+}$ induced $\mathrm{Ca}^{2+}$ release. J Physiol (Lond) 539:15-23.

Khimich D, Nouvian R, Pujol R, Tom Dieck S, Egner A, Gundelfinger ED, Moser T (2005) Hair cell synaptic ribbons are essential for synchronous auditory signalling. Nature 434:889-894.

Klyachko VA, Jackson MB (2002) Capacitance steps and fusion pores of small and large-dense-core vesicles in nerve terminals. Nature 418:89-92.

Lehnart SE, Huang F, Marx SO, Marks AR (2003) Immunophilins and coupled gating of ryanodine receptors. Curr Top Med Chem 3:1383-1391.

Lenzi D, Runyeon JW, Crum J, Ellisman MH, Roberts WM (1999) Synaptic vesicle populations in saccular hair cells reconstructed by electron tomography. J Neurosci 19:119-132.

Lindau M, Neher E (1988) Patch-clamp techniques for time-resolved capacitance measurements in single cells. Pflügers Arch 411:137-146.

Llano I, Gonzalez J, Caputo C, Lai FA, Blayney LM, Tan YP, Marty A (2000) Presynaptic calcium stores underlie large-amplitude miniature IPSCs and spontaneous calcium transients. Nat Neurosci 3:1256-1265.

Lollike K, Lindau M, Calafat J, Borregaard N (2002) Compound exocytosis of granules in human neutrophils. J Leukoc Biol 71:973-980.

Molina ML, Barrera FN, Fernandez AM, Poveda JA, Renart ML, Encinar JA, Riquelme G, Gonzalez-Ros JM (2006) Clustering and coupled gating modulate the activity in KcsA, a potassium channel model. J Biol Chem 281:18837-18848.

Moser T, Beutner D (2000) Kinetics of exocytosis and endocytosis at the cochlear inner hair cell afferent synapse of the mouse. Proc Natl Acad Sci USA 97:883-888.

Moser T, Neher E (1997a) Estimation of mean exocytic vesicle capacitance in mouse adrenal chromaffin cells. Proc Natl Acad Sci USA 94:6735-6740.

Moser T, Neher E (1997b) Rapid exocytosis in single chromaffin cells recorded from mouse adrenal slices. J Neurosci 17:2314-2323.

Neves G, Gomis A, Lagnado L (2001) Calcium influx selects the fast mode of endocytosis in the synaptic terminal of retinal bipolar cells. Proc Natl Acad Sci USA 98:15282-15287.

Nouvian R, Beutner D, Parsons TD, Moser T (2006) Structure and function of the hair cell ribbon synapse. J Membr Biol 209:153-165.
Oertner TG, Sabatini BL, Nimchinsky EA, Svoboda K (2002) Facilitation at single synapses probed with optical quantal analysis. Nat Neurosci 5:657-664.

Rohlich P, Anderson P, Uvnas B (1971) Electron microscope observations on compounds 48-80-induced degranulation in rat mast cells. Evidence for sequential exocytosis of storage granules. J Cell Biol 51:465-483.

Sakaba T (2006) Roles of the fast-releasing and the slowly releasing vesicles in synaptic transmission at the calyx of Held. J Neurosci 26:5863-5871.

Santos-Sacchi J (1991) Reversible inhibition of voltage-dependent outer hair cell motility and capacitance. J Neurosci 11:3096-3110.

Scepek S, Lindau M (1993) Focal exocytosis by eosinophils-compound exocytosis and cumulative fusion. EMBO J 12:1811-1817.

Sobkowicz HM, Rose JE, Scott GE, Slapnick SM (1982) Ribbon synapses in the developing intact and cultured organ of Corti in the mouse. J Neurosci 2:942-957.

Solsona C, Innocenti B, Fernandez JM (1998) Regulation of exocytotic fusion by cell inflation. Biophys J 74:1061-1073.

Spassova MA, Avissar M, Furman AC, Crumling MA, Saunders JC, Parsons TD (2004) Evidence that rapid vesicle replenishment of the synaptic ribbon mediates recovery from short-term adaptation at the hair cell afferent synapse. J Assoc Res Otolaryngol 5:376-390.

Sun JY, Wu XS, Wu LG (2002) Single and multiple vesicle fusion induce different rates of endocytosis at a central synapse. Nature 417:555-559.

Takahashi N, Hatakeyama H, Okado H, Miwa A, Kishimoto T, Kojima T, Abe $\mathrm{T}$, Kasai H (2004) Sequential exocytosis of insulin granules is associated with redistribution of SNAP25. J Cell Biol 165:255-262.

Takamori S, Holt M, Stenius K, Lemke EA, Gronborg M, Riedel D, Urlaub H, Schenck S, Brugger B, Ringler P, Muller SA, Rammner B, Grater F, Hub JS, De Groot BL, Mieskes G, Moriyama Y, Klingauf J, Grubmuller H, Heuser J, Wieland F, Jahn R (2006) Molecular anatomy of a trafficking organelle. Cell 127:831-846.

Tong G, Jahr CE (1994) Multivesicular release from excitatory synapses of cultured hippocampal neurons. Neuron 12:51-59.

von Gersdorff H, Matthews G (1994) Dynamics of synaptic vesicle fusion and membrane retrieval in synaptic terminals. Nature 367:735-739.

von Gersdorff H, Vardi E, Matthews G, Sterling P (1996) Evidence that vesicles on the synaptic ribbon of retinal bipolar neurons can be rapidly released. Neuron 16:1221-1227.

Wadiche JI, Jahr CE (2001) Multivesicular release at climbing fiberPurkinje cell synapses. Neuron 32:301-313.

Wu XS, Xue L, Mohan R, Paradiso K, Gillis KD, Wu LG (2007) The origin of quantal size variation: vesicular glutamate concentration plays a significant role. J Neurosci 27:3046-3056. 